\title{
SEROEPIDEMIOLOGÍA DE LA RINOTRAQUEITIS INFECCIOSA BOVINA EN EL MUNICIPIO DE MONTERÍA, COLOMBIA
}

\author{
SEROEPIDEMIOLOGY OF THE IN FECTIOUS BOVINE RHINOTRACHEITIS \\ IN THE MUNICIPALITY OF MONTERÍA, COLOMBIA
}

\author{
César Betancur H*, Marco G onzález T, Lázaro Reza G. \\ Universidad de Córdoba, Facultad de Medicina Veterinaria y Zootecnia. Departamento de Ciencias Pecuarias. \\ Montería, Colombia.*Correspondencia: betanci@ yahoo.com
}

Recibido: Febrero 27 de 2006; Aceptado: Junio 1 de 2006

\section{RESUMEN}

O bjetivo. Estudiar la sero prevalencia de IBR en ganado bovino con antecedentes de infertilidad. Materiales y métodos. Se recolectaron 150 muestras de sangre de hembras con antecedentes de infertilidad y sin historia de vacunación contra IBR, pertenecientes a 32 fincas distribuidas en el municipio de Montería. Adicionalmente, se obtuvieron muestras al azar de 20 toros pertenecientes a las mismas fincas. Se analizaron anticuerpos contra IBR mediante la prueba de ELISA. Resultados. Los resultados mostraron una seroprevalencia de $74.7 \%$ para rinotraqueitis infecciosa bovina. Se encontraron diferencias significativas en cuanto a prevalencía para las variables sexo y edad de los animales $(p<0.05)$. No hubo diferencias significativas en prevalencía para las variables raza, zona, tipo de explotación y alteraciones reproductivas $(p>0.05)$. Conclusión. La alta prevalencia de la infección por IBR en vacas podrían correlacionarse con la infección en toros, lo cual tiene un significado relevante, ya que la infección es de transmisión venérea. Estos resultados deben alertar a las autoridades sanitarias para que implementen estrategias de prevención y control.

Palabras Clave: Rinotraqueitis infecciosa bovina (IBR), infección herpesviral, bovinos, infertilidad bovina, Colombia.

\section{ABSTRACT}

O bjective. To study the seroprevalence of IBR in livestock bovine with infertility antecedents. Materials and methods. Blood samples were collected from 150 animals with infertility antecedents and nonvaccinated history against IBR, belonging to 32 farms in Monteria municipality and samples at random of 20 bulls belonging to the same farms. Antibodies were analyzed against IBR by using ELISA test. Results. The results showed a seroprevalence of $74.7 \%$ for bovine rhinotracheitis infectious. They were significant differences with regard to prevalence for sex and age variables of animals $(p<0.05)$. There were not significant differences in prevalence for the variable race, area, type of exploitation and reproductive alterations $(p>0.05)$. Conclusion. The high prevalence of the infection for IBR in cows could be associated with the infection in bulls, which has an outstanding meaning, 
since the infection is of venereal transmission. These results should alert to sanitary authorities in order to implement prevention and control strategies.

Key words: Infectious bovine rhinotracheitis (IBR), herpes viral infectious, bovine infertility, Colombia.

\section{INTRODUCCIÓN}

La rinotraqueitis infecciosa bovina (IBR) es producida por el herpesvirus bovino tipo HVB-1, clasificado dentro de la familia herpesviridae, subfamilia alfa herpesvirinae; posee cubierta lipidica, capside icosahedrica y ácido nucleico ADN (1). Múltiples copias del ADN viral pueden permanecer como epitomas integrados al genoma celular de las neuronas de los ganglios nerviosos en donde establecen infecciones latentes (2).

Se han descrito 2 subtipos de BHV-1: BHV-1 subtipo 1 representan cepas que causan enfermedad respiratoria: rinotraqueitis infecciosa bovina (IBR); mientras el subtipo 2 incluye cepas que causan enfermedad genital, como Vulvovaginitis Pustular Infecciosa (VPI) y Balanopostitis Infecciosa (BPI) (3).

La forma genital conocida VPI-BPI es una infección venérea caracterizada por infertilidad temporal $(4,5)$. 0 tras presentaciones incluyen dermatitis, mastitis y metritis (6 - 8) y la forma encefálica descrita como una enfermedad altamente mortal en terneros (9).

El aborto es otra de las manifestaciones importantes de esta enfermedad; el feto es susceptible a la infección durante todo el perío do de gestación pudiendo causar la muerte aún dentro de las primeras horas de vida. Aunque el aborto es más que una secuela del problema respiratorio, hay reportes de cepas con cierto potencial abortigénico que pueden producir brotes de abortos (5). En vacas lactantes, produce disminución de la producción (10) e infertilidad (11).

El virus se transmite en forma directa por aerosoles o por contacto con animales infectados, a partir de secreciones respiratorias, oculares y del tracto reproductivo, o en forma indirecta a través de personas o equipos. También se puede transmitir a través del semen, bien sea por monta natural o por inseminación artificial $(12,13)$ e incluso durante la transferencia de embriones $(14,15)$.

Uno de los mayores problemas en el control de la infección del HVB-1 es la capacidad del virus de permanecer en estado latente y persistir así por largos períodos de tiempo o reactivarse periódicamente, como consecuencia de estrés fisiológico del animal o por tratamiento con corticoides (16). Los animales con infección latente son usualmente identificados por la detección de anticuerpos específicos contra el HVB-1 en muestras de suero (17).

En Colombia se ha reportado para hembras bovinas una seroprevalencia del $51.7 \%$ para la región Caribe, un $21.5 \%$ para la región Andina y un $20.6 \%$ para el pie de monte llanero (18). En toros de la sabana de Bogotá se encontró un $15.3 \%$ de reactores positivos; sin embargo, son pocos los aislamientos virales (19). En un informe de 2952 muestras de suero bovino, procedentes de diferentes regiones del país, por la técnica de ELISA, se reportó un $53.4 \%$ de positividad a IBR (observaciones no publicadas). En un estudio realizado en los departamentos de Córdoba y Sucre, entre los años 1980 1984 , se encontró una prevalencia del $29.6 \%$ en muestras de suero provenientes de 2295 bovinos (20). El estudio demostró que no existieron diferencias significativas entre el ganado lechero y el de carne y que los índices de prevalencia aumentaron progresivamente conforme aumentó la edad de los animales (20).

El objetivo del presente trabajo fue el de determinar la prevalencia de anticuerpos contra IBR en bovinos con antescedentes de infertilidad.

\section{MATERIALES Y MÉTODOS}

Sitio de estudio. El trabajo se desarrolló en el municipio de Montería, departamento de Córdoba, cuya temperatura promedio anual es 
de $28^{\circ} \mathrm{C}$, humedad relativa del $85 \%$ y una precipitación anual de 1200 a $1500 \mathrm{~mm}$. El área municipal es de $3.043 \mathrm{Km}^{2}$, conformado por 27 corregimientos, limita al Norte con Puerto Escondido, San Pelayo y Cereté, al Este con San Carlos y Planeta Rica, al Sur con Tierralta y Valencia y al 0 este con el departamento de Antioquia y Canalete (21). El municipio de Montería cuenta con una población bovina de 489.036 bovinos de los cuales 178.320 son hembras mayores de 2 años (22).

Tipo de estudio y tamaño de la muestra. El estudio fue de tipo descriptivo y transversal. El tamaño de la muestra se determinó mediante el programa estadístico EPI-IN FO, 2000, versión 6.0 CDC, Atlanta, GA, USA y correspondió a 108 animales, sin embargo, en el presente estudio se utilizaron 170 sueros, provenientes de 32 fincas del municipio de Montería. Para la interpretación de los datos estadísticos se usó un nivel de significancia del $5 \%$.

Selección de la muestra. Como criterios de inclusión se tuvieron en cuenta las hembras repetidoras (más de tres servicios sin gestación) y/o que tuvieron reporte de abortos (tempranos o tardíos) y no tuvieran historia de vacunación. El estudio fue realizado a través de las evaluaciones reproductivas rutinarias que se adelantaron en la explotación. En cada finca se tomaron muestras a 5 hembras que cumplían por lo menos uno de los criterios de inclusión y por lo menos 1 toro; se obtuvo información referente a identificación del animal edad, raza, tipo de explotación y estado reproductivo, variables necesarias para la correlación con la seropositividad a IBR.

O btención del suero y procesamiento de las muestras. Previa desinfección de la zona se colectaron $10 \mathrm{ml}$ de sangre en la vena yugular o coccígea. La sangre fue recogida en tubos tipo vacutainer ${ }^{\circledR}$ sin anticoagulante para obtener el suero por métodos estándares conocidos. El procesamiento de las muestras se realizó en el laboratorio de microbiología IIBT de la Universidad de Córdoba y las determinaciones de IBR fueron realizadas en la Universidad Nacional de Colombia mediante la prueba de ELISA indirecta. Las lecturas de densidad óptica y sus valores se tradujeron a porcentajes con puntos de corte establecidos en 0.150 donde cualquier resultado que estuvo por encima de este límite se consideró positivo.

Análisis esta dístico. Se utilizaron la distribución Ji cuadrado y los coeficientes de contingencia de Pearson y Gamma, para analizar el grado de asociación entre las distintas variables. Para la obtención de los resultados de las distintas variables se utilizó el paquete estadístico SPSS versión 11 (23).

\section{RESULTADOS Y DISCUSIÓN}

El promedio de los títulos de anticuerpos para IBR en las fincas muestreadas fue del 74,7\% (Figura 1). Estos resultados se encuentran por encima de valores hallados en otros trabajos realizados en el país, cuyas cifras estuvieron desde $20.6 \%$ hasta $53.4 \%$ (18 - 20).

En este trabajo se incluyeron 20 toros, de los cuales 19 resultaron positivos (95\%), lo que implica que los resultados tienen un significado relevante por tratarse de una infección latente y de transmisión venérea.

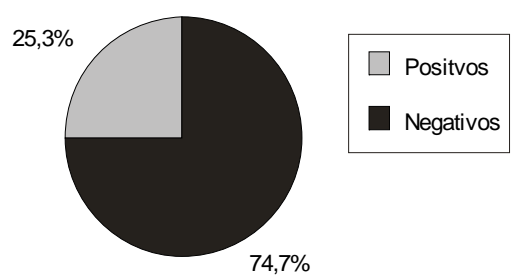

Figura 1. Porcentaje de animales positivos y negativos a IBR.

Análisis del factor edad. En el grupo de animales con edades entre los 3 y 4 años se encontró un $36.1 \%$ de hembras positivas a IBR, mientras que entre 5 y 6 años este fue del $46.3 \%$ y en los hombres de 70 más años $17.6 \%$. (Figura 2). La segunda situación pudo deberse a que eran animales que se encontraban en la fase productiva más exigente, lo cual expone a las vacas a una serie de factores estresantes que inmunosuprimen a los animales, lo que podrían facilitar la puerta de entrada a diversos agentes infecciosos (24).

El valor ji-cuadrado de la prueba de independencia fue de 6.20 que resulta significativo con un $p$-valor de $0.045(p<0.05)$, 
dando cierta dependencia entre estas dos variables. El grado de asociación, entre edad e IBR, medido a través del coeficiente Gamma, fue del $19.1 \%$.

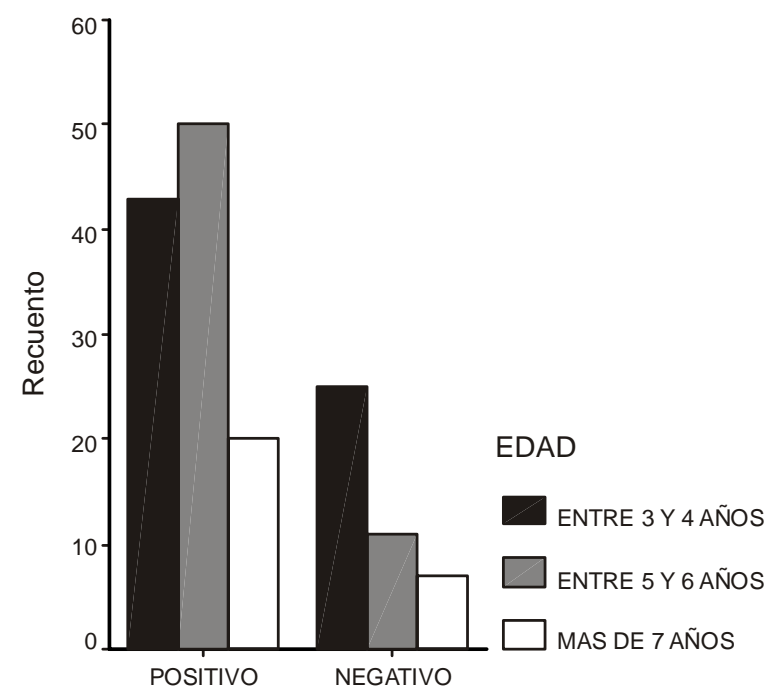

Figura 2. Grado de asociación entre IBR y la edad de las hembras.

Análisis del factor raza o cruce. La representación de la raza o cruce en la muestra está dada ante todo por la mestiza $(48.8 \%)$ y la cebuína (47.1\%); el resto fue de tipo europeo (Figura 3). Al analizar el grado de asociación entre IBR con respecto a la raza se encontró un valor del coeficiente de contingencia de Pearson

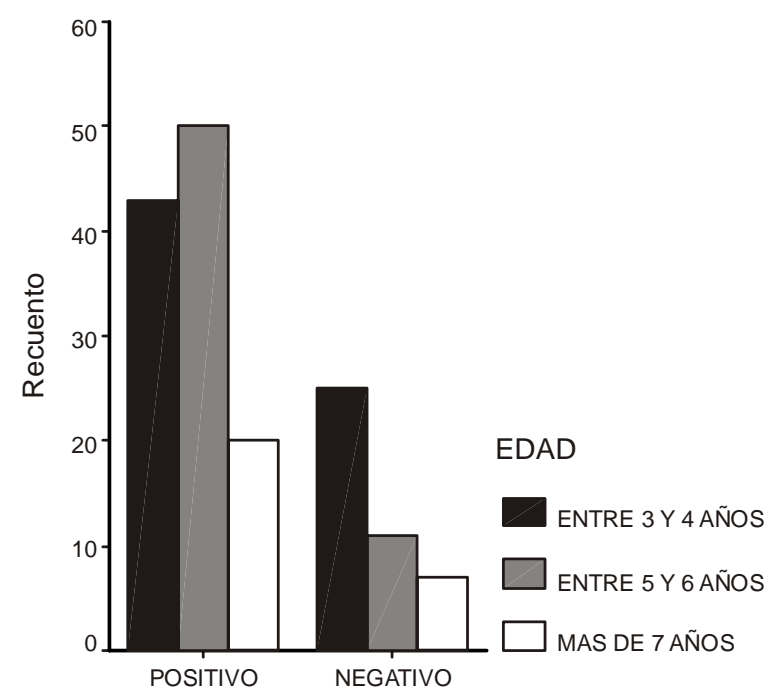

Figura 3. Grado de asociación IBR-raza de $10.5 \%$ y un valor de ji-cuadrado de 1.96 , el cual no es significativo estadísticamente $(p>0.05)$. Por lo que se puede afirmar que la presencia de esta enfermedad, no está influenciada por la raza o cruce del animal.

Análisis de la zona. El área de estudio se dividió en cuatro zonas, norte, sur, este y o este (Figura 4). En la zona se presentó mayor seroprevalencia de IBR (29.6\%) seguido por la zona norte $(25.9 \%)$, lo cual puede estar correlacionado con mayor movilidad de ganado en estas áreas. Sin embargo, el coeficiente de correlación (Pearson) de IBR con la variable zona, fue del $13 \%$, resultando no significativo, con un valor de ji-cuadrado de 4.09 ( $p$-valor $=0.25)$ lo que sugiere que dicha enfermedad se puede presentar indistintamente en cualquiera de las zonas estudiadas.

Análisis del factor: tipo de explotación. El $65.9 \%$ de los animales estudiados eran de explotación doble propósito y el resto de cría libre. Se observó una mayor frecuencia de presentación de IBR (67.6\%) en las explotaciones de doble propósito que en las de cría libre. No obstante, el grado de asociación entre estas dos variables tan sólo alcanzó el $5.4 \%$ resultando no significativa ( $p$-valor $=0.38$ ).

Esto concuerda con las afirmaciones hechas por otros autores, quienes expresaron que la transmisión de IBR vía genital es predominantemente en las ganaderías extensivas independientemente del tipo de explotación y por ende del ganado (20).

Análisis del estado reproductivo. Al comparar la prevalencia de IBR entre vacas repetidoras y con antecedentes de aborto se encontró que el $74 \%$ de las primeras resultaron positivas mientras que el $70 \%$ de las segundas también tuvieron este mismo resultado, es así como el coeficiente de asociación entre estas dos variables fue de sólo el $4 \%$, el cual resulto no significativo ( $p$-valor $=0.623$ ). Esto sugiere que la presencia de esta enfermedad de acuerdo con la condición reproductiva es independiente, teniendo igual probabilidad de presentación para cualquiera de los estados reproductivos. 


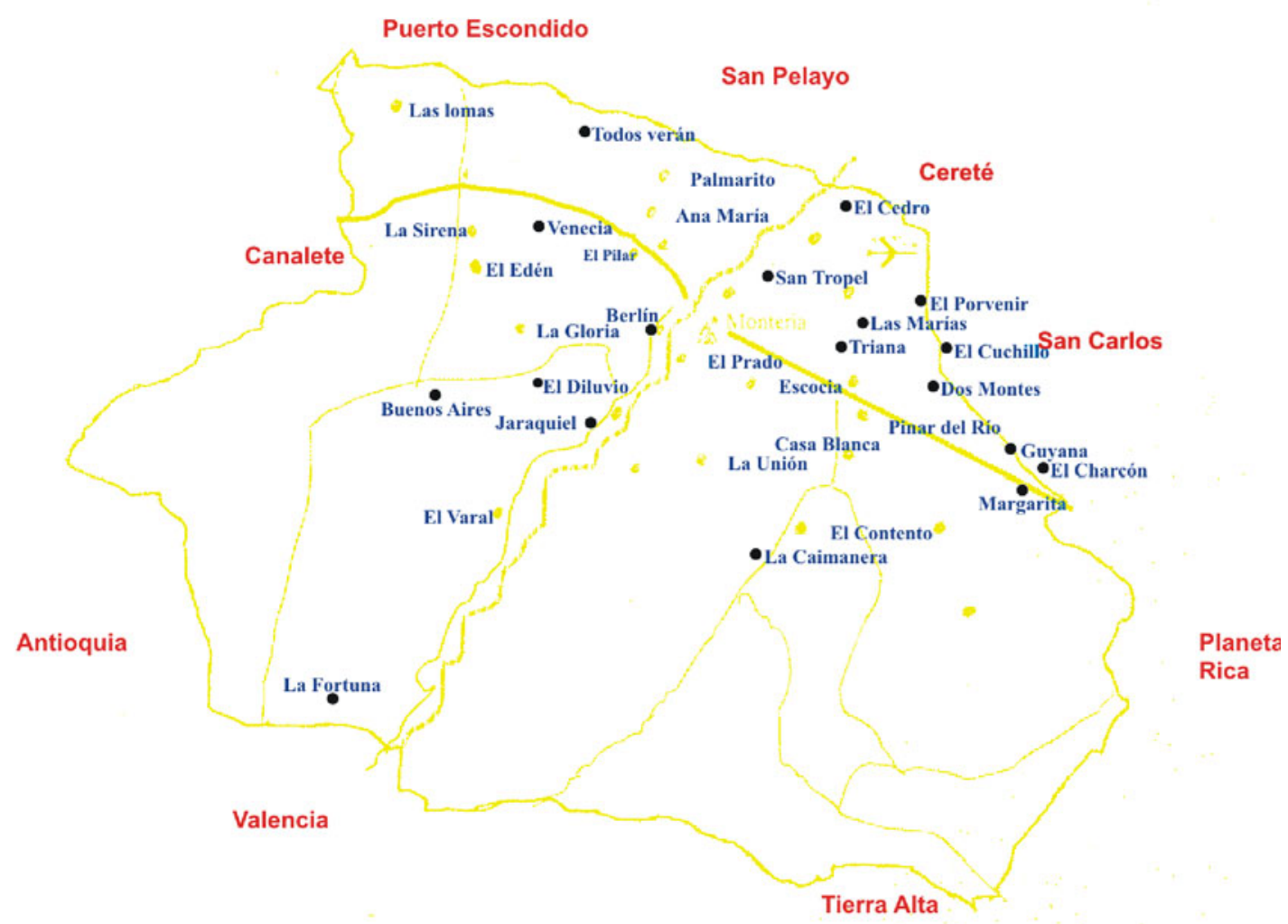

Figura 4. Municipio de Montería indicando las cuatro zonas de muestreo

\section{Influencia del toro. Asociación:} sexo-IBR.

La investigación también arrojó algunos resultados concernientes a la influencia del toro con relación a la infección en las vacas. En este sentido se encontró que existe una dependencia entre la presencia de IBR con respecto al sexo, es así como el $72 \%$ de las hembras y el $95 \%$ de los toros resultaron positivos con IBR (Figura 5). El valor ji-cuadrado fue de 4.94 con un coeficiente de correlación de Pearson de $16.8 \%$, con un $p$-valor $=0.027$ $(p<0.05)$, encontrándose así diferencias estadísticas en esta enfermedad con respecto al sexo. Por lo tanto, los toros infectados podrían ser una importante fuente de transmisión de IBR; resultados que concuerdan con Eskra et al (25) quienes comprobaron que en los toros la forma latente del virus puede jugar un papel importante en la difusión de la enfermedad.

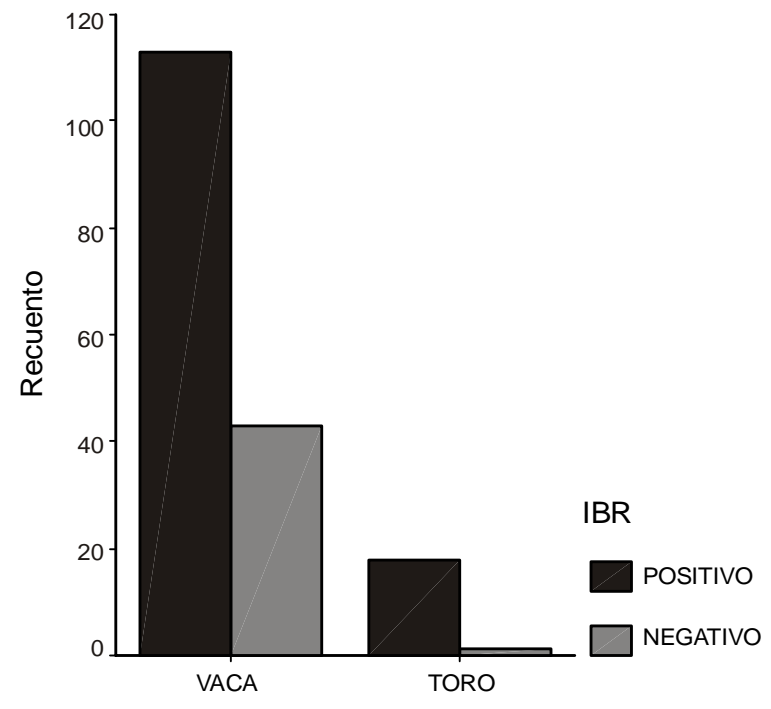

Figura 5. Asociación Sexo - IBR. 
En conclusión, la alta prevalencia de la infección por IBR en vacas podrían correlacionarse con la infección en toros, 10 cual tiene un significado relevante, ya que la infección es de transmisión venérea. Estos resultados deben alertar a las autoridades sanitarias para que implementen estrategias de prevención y control.

\section{Agradecimientos}

A la oficina Administradora de Investigación y Extensión de la Universidad de Córdoba que financió este proyecto; Laboratorio Novartis de Colombia S.A., al Laboratorio de Microbiología de la Universidad Nacional, al laboratorio de Microbiología y al IIBT de la Universidad de Córdoba. A Bartolo Petro.

\section{REFERENCIAS}

1. Ackermann M, Engels M. Pro and contra IBR-eradication. Vet Microbiol 2006; 113: 293-302.

2. Hammerschmidt W, Lurz R, Ludwig H, Buhk $H J$. Recombination of genomic terminus of bovine herpesvirus-1 with cellular DNA. J Gen Virol 1990; 71: 2043-2051.

3. Radostits OM, G a y CC, Blood DC, Hinchcliff KW. Veterinary Medicine: $A$ textbo ok of the Diseases of $C$ attle, Sheep, Pigs, Goats and Horses, 9 th. W.B. Saunders Company Ltda. 2000; p.1173-1184.

4. Babiuk LA, L'italien J, Den Hurk SDL. et al. Protection of cattle from bovine herpesvirus type 1 (BHV-1) infection by immunization with individual viral glycoproteins. Virology 1987; 159: 57-66.

5. Miller J, C Whetstone, M Van Der Maten. Abortifacient potencial of bovine herpesvirus tipo 1 isolates that represent three subtypes determined by restriction endonuclease análisis of viral DNA. Am J Vet 1991; 52: 458-461.

6. Bwagamoi 0 , Kaminjolo JS. Isolation of IBR/IPV virus from semen and skin lesions of bulls at Kabete, Kenya. Zentralbl Veterinarmed . 1971; 18: 262-269.

7. G reig AS, Bannister GL. Infection of the bovine udder with bovine herpesvirus. Can J Comp Med Vet Sci 1965; 29: 57-62.
8. Lomba F, Bienfet $\mathrm{V}$, Wellemans $\mathrm{G}$. IBR virus and occurrence of metritis at partutition in the bovine Belgian blue white breed. Br Vet J 1976; 132: 178-181.

9. G eorge LW. Understanding the encephalitic form of infectious bovine rinotracheitis. Food Anim Pract 1991; 335-337.

10. Straub OC. Infection bovine rhinotracheitis virus. Virus Inf Ruminant 1990; 3: 71-108.

11. Trapp S, Beer M, M ettenleiter TC. Biology of bovine herpesviruses. Berl Munch Tierarztl Wochenschr 2003; 116: 171178.

12. Wiedmann M. Detection of bovine herpesvirus- 1 in bovine semen by a nested PCR assay. J Virol Methods 1993; 44.

13. Van 0 irschot J. Bovine herpesvirus 1 in semen of bulls and the risk of transmission: a brief review. Vet Q 1995; 17: 29-33.

14. Wentink G, Van O irschot J, Verhoeff J. Risk of infection with bovine herpes virus 1 (BHV-1): a review. Vet Q 1993; 15: 3033.

15. Engels M, Ackermann M. Pathogenesis of ruminant herpesvirus infections. Vet Microbiol 1996; 53: 3-15.

16. Whetstone CA, Miller JM, Bortner DM, Van der Maaten MJ. Changes in the bovine herpesvirus 1 genome during acute infection, after reactivation from latency 
and after superinfection in the host a nimal. Arch Virol 1989; 106: 261-279.

17. Lemaire $M$. Production of bovine herpesvirus type 1 seronegative latent carriers by administration of a live attenuated vaccine in passively immunized calves. J Clin Microbiol 2000; 38: 11.

18. Griffiths IB, Gallego MI, Villamil LC. Factores de infertilidad y pérdidas económicas en ganado de leche en Colombia. Publicaciones ICA. 1982; p.168.

19. Góngora A, Villamil LC, Vera V, Ramírez $G$, Parra J. Diagnóstico de las principales enfermedades reproductivas en toros de la Sabana de Bogotá. Énfasis en RIB. Rev Med Vet Zoot 1995; 43: 37-41.

20. $O$ tte $E, N$ avarrete $M, O$ rjuela J. Resultados de una encuesta realizada sobre producción y salud animal en MonteríaCórdoba, Colombia. 1982-1984: parte II. Proyecto Colombo Alemán ICA-GTZ. Informe técnico. 1985; p.1-125.
21. IGAC Instituto Geográfico Agustín Codazzi. Seccional Montería. Comunicación institucional. 2006.

22. ICA FEDEGAN. Informe censo bovino II ciclo de vacunación Aftosa- Brucelosis. Comunicación institucional. 2005.

23. Siegel S. Estadística no paramétrica aplicada a las ciencias de la conducta. Editorial trillas, M éxico. 2005.

24. G onzález C. Influencia de diversos factores sobre la duración de la gestación en el ganado de lidia. (Publicación en línea) 2003. Disponible en: http:// www.uclm.es/ profesorado/produccionanimal/ Artículostaurinos/ar.pdf. (acceso 15/11/ 2004).

25. Eskra L, Splitter GA. Bovine herpesvirus-1 infects activated CD4 (+) lymphocytes. J Gen Virol 1997; 78: 2159-2166. 